\title{
Demonstration of on sky contrast improvement using the Modified Gerchberg-Saxton algorithm at the Palomar Observatory
}

\author{
Rick S. Burruss* ${ }^{\mathrm{a}}$, Eugene Serabyn ${ }^{\mathrm{a}}$, Dimitri P. Mawet ${ }^{\mathrm{a}}$, Jennifer E. Roberts ${ }^{\mathrm{a}}$, Jeffrey P. Hickey ${ }^{\mathrm{b}}$, \\ Kevin Rykoski ${ }^{\mathrm{b}}$, Siddarayappa Bikkannavar ${ }^{\mathrm{a}}$, Justin R. Crepp ${ }^{\mathrm{c}}$ \\ ${ }^{a}$ Jet Propulsion Laboratory, California Institute of Technology, Pasadena, CA, USA 91109; \\ ${ }^{b}$ Palomar Observatory, Palomar Mountain, CA, USA 92060-0200; \\ ${ }^{\mathrm{c}}$ California Institute of Technology, Pasadena, CA USA 91125
}

\begin{abstract}
We have successfully demonstrated significant improvements in the high contrast detection limit of the Well-Corrected Subaperture (WCS) using a number of steps aimed at reducing non-common path (NCP) wavefront errors, including the Autonomous Phase Retrieval Calibration (APRC) ${ }^{1}$ software package developed at the Jet Propulsion Laboratory (JPL) for the Palomar adaptive optics instrument (PALAO). APRC utilizes the Modified Gerchberg-Saxton (MGS) wavefront sensing algorithm, also developed at $\mathrm{JPL}^{2}$. The WCS delivers such excellent correction of the atmosphere that NCP wavefront errors not sensed by PALAO but present at the coronagraphic image plane begin to factor heavily as a limit to contrast. The APRC program was implemented to reduce these NCP wavefront errors from $110 \mathrm{~nm}$ to $35 \mathrm{~nm}$ (rms) in the lab, and now these exceptional results have been extended to targets on the sky for the first time, leading to a significant suppression of speckle noise. Consequently we now report a contrast level of very nearly $1 \times 10^{-4}$ at separations of $2 \lambda / \mathrm{D}$ before the data is post processed, and $1 \times 10^{-5}$ after post processing. We describe here the major components of our instrument, the work done to improve the NCP wavefront errors, and the ensuing excellent on sky results, including the detection of the three exoplanets orbiting the star HR8799.
\end{abstract}

Keywords: phase retrieval, mgs, high contrast coronagraphy, wavefront sensing, adaptive optics, HR8799

\section{INTRODUCTION}

Directly imaging exoplanets orbiting very bright stars exposes significant technical challenges. The three exoplanets orbiting HR8799 were recently directly discovered using differential imaging on a large telescope ${ }^{3}$. Scattered star light emanating from uncorrected wavefront errors limit the contrast between star light and exoplanet light. These wavefront errors also push the detectable region where exoplanets can be seen further and further away from the host star. Depending on the chosen mask, most modern coronagraphs start to reach noteworthy contrast levels at angular separations from the center of the host star of about $10 \lambda / D$ (where $\lambda$ is the observing wavelength and $D$ is the aperture diameter), giving large telescopes a distinct advantage to exoplanet detection. To detect faint stellar companions at smaller angular offsets, wavefront errors present in the imaging system need to be significantly reduced, while efficiently blocking the star light and allowing the companion light to pass to the detector.

The residual wavefront errors that lead to the speckle noise which degrades contrast emerge from several sources. The adaptive optics instrument that delivers the wavefront to the science camera has a finite ability to correct for the turbulence in the atmosphere. Residual errors from this correction lead to speckles in the image which look like the planets we are trying to detect. In addition, errors at the science camera not sensed by the adaptive optics wavefront sensor camera contribute to the speckle noise. These NCP errors include differential image drift between the two cameras, differential focus between the cameras, and a quasi-static residual error due to small errors in the science arm induced primarily by flexure, brought on both by temperature gradients and changing gravity loads on the science arm as the telescope position is changed.

Adaptive Optics Systems II, edited by Brent L. Ellerbroek, Michael Hart, Norbert Hubin, Peter L. Wizinowich, Proc. of SPIE Vol. 7736, 77365X - ๔ 2010 SPIE · CCC code: 0277-786X/10/\$18 - doi: 10.1117/12.857544 
Our group initiated an effort to reach the $1 \times 10^{-5}$ contrast milestone at $\lambda / \mathrm{D}$ angular separations using the existing WCS, PALAO, and Palomar infrared science camera (PHARO). To effectively block the star light and allow for exoplanet detections very close to the host star the novel vector vortex coronagraphic (VVC) phase mask was added to the PHARO focal plane selector mechanism. To minimize image drift of the star from behind this mask during integrations new actuator mounts were added to the PALAO instrument. To reduce the NCP quasi-static wavefront errors we chose to incorporate the excellent APRC software package developed at JPL and implemented at Palomar Observatory in the lab in 2007. At that time APRC had only been demonstrated in the lab. We present here the work done to extend the APRC lab results to the sky, and also note the improvements gained by the new star selector mounts, both of which are now available to all PALAO users. This work, coupled with the excellent performance of both the VVC mask and effective post processing techniques, led to the detection of the three exoplanet companions of HR8799.

\section{THE WCS INSTRUMENT}

The WCS instrument reimages a clear, 1.5 meter diameter, off-axis subaperture of the Palomar Hale telescope and delivers this reimaged wavefront to the PALAO instrument ${ }^{4}$. The resulting residual wavefront errors then passed on to the science detector are reduced from 200-250 nm rms when using the full telescope to $85-100 \mathrm{~nm}$ rms when using WCS. The PHARO near infrared camera was used for this work, and Palomar Observatory has graciously allowed the addition of our image and pupil plane masks to this camera. Note that any PALAO science camera can take advantage of the excellent correction provided by the WCS. The four-quadrant phase mask was used for the first high contrast experiments with the WCS and PHARO $^{5}$. While the resulting correction of the atmosphere was excellent, even providing suitable correction for visible images in the B-band, the speckle noise due to NCP wavefront errors limited the contrast ratio to about $10^{-3}$. Image drift and target centering during these experiments was also a problem. Due to NCP flexure between PALAO and PHARO, targets drifted by 10 to 20 milliarcseconds (mas) per minute, requiring constant target position updates with mirror actuators whose smallest allowable offset was 22 mas steps. The uni-directional accuracy of these mounts was measured at 3 mas for commanded moves above 22 mas. Backlash of these mounts was a serious problem, so much so that if the target motion direction was changed at all during an observation, the entire alignment process had to be repeated.

\subsection{THE VECTOR VORTEX CORONAGRAPH}

A K-short band $(2.15 \mu \mathrm{m}) \mathrm{VVC}$ phase mask was installed in the PHARO focal plane to block the bright light of host stars $^{6}$. We prefer phase masks because they are transparent and can transmit exoplanet light at small angular separations from the host star. The VVC mask's theoretical half-power transmission occurs at 0.9 $\lambda / \mathrm{D}$ from the center (about 260 mas in this case). A stringent tolerance on pointing and drift exists with these masks however, and needs to be accounted for. For example, given suitable wavefront correction at the focal plane where the mask resides, the VVC requires pointing to the center of the mask to be better than $0.02 \lambda / \mathrm{D}$ (about 5 mas in this case). 
The vortex coronagraph

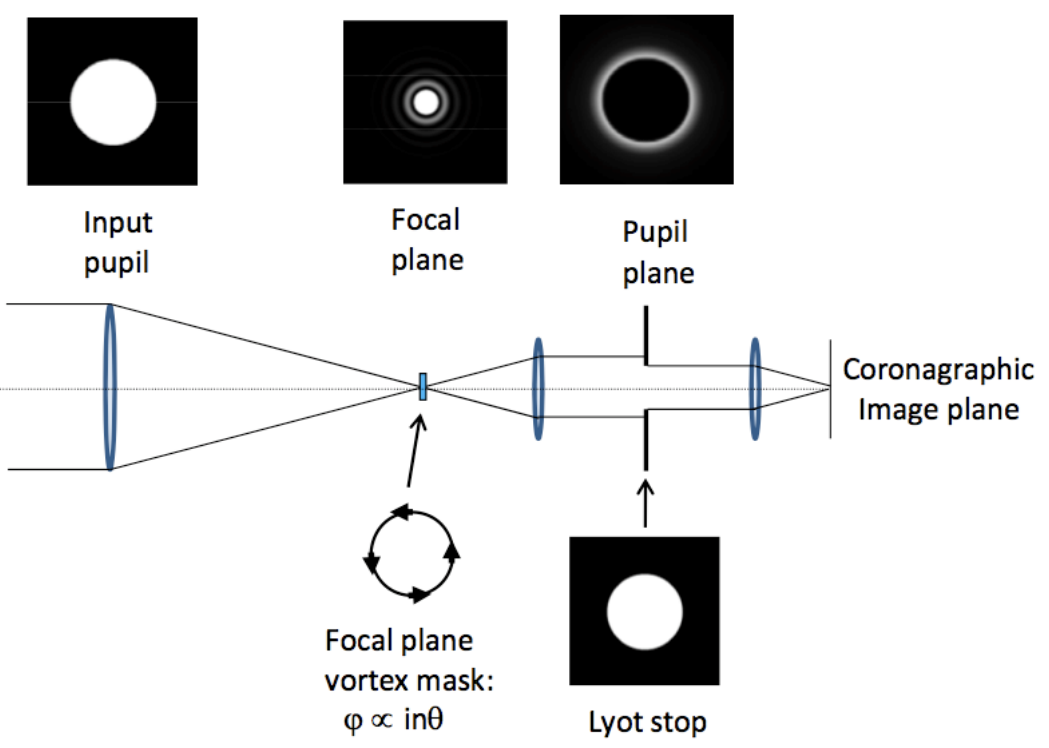

Figure 1. Conceptual layout of the Vector Vortex coronagraph: The light from the PALAO input pupil is focused onto the PHARO focal plane coronagraphic phase mask. The azimuthal phase ramp is induced by traveling through a rotationally symmetric half-wave plate. The output phase, $\phi$, is linearly proportional to the azimuthal angle, $\theta$. The beam is recollimated after the VVC. In an ensuing PHARO pupil plane, an opaque undersized pupil stop (Lyot stop) blocks the starlight which, for a perfect wavefront, lies entirely outside the image of the original pupil.

\subsection{PALAO IMAGE POINTING MOUNTS}

To solve the pointing and drift problems, custom Aerotech ${ }^{8}$ gimbal mounts were purchased and installed to actuate the PALAO image pointing optics, replacing gimbal mounts that had been in service for nearly ten years. These dualencoded, motorized gimbal mounts were made to fit into the allowable space on the optical bench. Testing showed a significant improvement in both image positioning and image drift caused by flexure. A bug was also discovered in the motor control software that nullified moves less than 22 mas. After fixing this bug, uni-directional image position accuracy is now better than 2 mas. Bi-directional image position accuracy (backlash) is better than 3 mas. This excellent image positioning performance allows targets to be centered on the mask quickly, and allows for extremely accurate image drift compensation using small, accurate image motion commands.

Further, image drift due to flexure was vastly improved once these mounts were installed. Image drift using the new mounts is now measured at less than 1 to 2 mas per minute, depending on telescope position, significantly improving observing efficiency. Science target acquisition has been reduced to a few minutes, as opposed to several tens of minutes. And once the target is centered on the VVC mask, keeping it there requires fewer target position updates since the NCP image drift has been so dramatically reduced. When observing on sky, the residual light scattered around the VVC mask in science images is monitored as they are displayed, which appears like a "doughnut" of light around the mask. At most telescope pointings where the image drift is less than 1 mas per minute, small, accurate corrections to the new mounts are issued once every 100 seconds to keep the target centered to within the 5 mas centering requirement of the VVC. A software algorithm is now being implemented to monitor the light leak from behind the VVC in PHARO science images, and send image motion commands to PALAO to automatically keep targets centered on the VVC mask. 


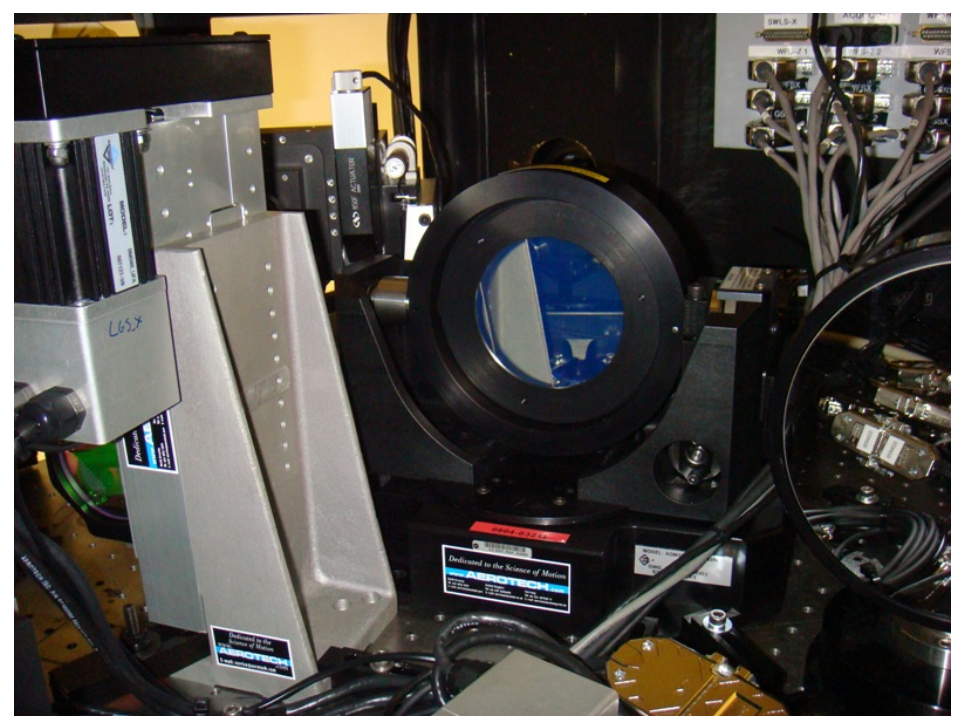

Figure 2. Custom Aerotech dual encoded gimbal mount: Star Selector Mount \#1 in the PALAO instrument, which holds the science camera dichroic filter. The infrared light passes through this filter, while the visible light reflects to a mirror held in the other Aerotech gimbal mount. These two mounts are commanded in tandem to accurately move targets in both the PALAO and science instruments.

\section{APRC UPDATES}

APRC uses the PHARO science camera as the NCP wavefront measurement device. Two defocused images on either side of the camera's focal plane are taken of the internal white light source by moving the white light source stage and commanding PHARO to record images. These images are then sent to JPL for the MGS processing. The MGS result is an estimate that is converted into DM actuator voltages. These DM maps are then sent back to Palomar, applied to the $\mathrm{DM}$, and the process is repeated until the NCP error is minimized. The entire process is done open loop with no high order correction applied. In 2007 APRC corrected the NCP residual error from $110 \mathrm{~nm}$, which is the best that can be done by hand tuning, to below $40 \mathrm{~nm}$ rms. Due to complications converting these maps into the proper form that PALAO can use when the high order loops are closed, the MGS results were not used on the sky.

In 2008 the PALAO optical bench was realigned to allow for larger science instruments. In addition to this realignment, a new all-reflective stimulus package containing a broadband white light source and alignment laser was installed to replace the original stimulus sources. Due to these changes to the optical path, a new set of APRC influence measurements was needed before APRC could move forward. Also, PALAO and PHARO had undergone several control software updates since APRC was first implemented. Communication and security protocols between Palomar and JPL had also changed. All of these issues were addressed in an attempt to streamline the APRC process into something that the Palomar night staff could use efficiently as a general PALAO observing tool.

\subsection{APRC INFLUENCE MEASUREMENTS}

Due to several changes and upgrades to both PALAO and PHARO control software, a few APRC revisions were necessary to regain communication to the hardware in the lab. Several communication issues between Palomar and JPL that had arisen due to security upgrades at Palomar were fixed. After this minimal effort to resurrect the APRC scripts, APRC was correctly measuring the NCP wavefront error in the lab. 

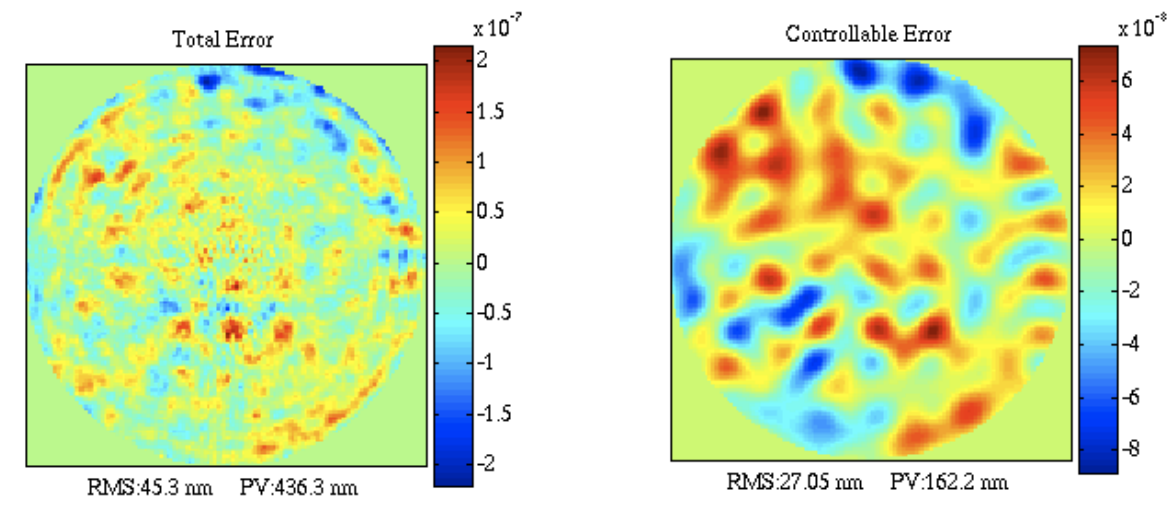

Figure 3. Preliminary APRC lab results: left. The total APRC residual error estimate after 4 iterations in February 2009 before any updates. The final residual NCP wavefront error is $45 \mathrm{~nm}$ rms right. The controllable APRC residual error estimate after the same 4 iterations. The controllable estimate is generated by passing the total error through a low-pass filter at 8 cycles per pupil. This is the residual error that the PALAO DM should be able to correct.

Next, using the APRC influence measurement tools developed in 2007, the influence functions of the DM were remeasured by poking DM actuators equal amounts in a grid pattern. A standard set of defocused PHARO images of these grid patterns was then taken and MGS was used to measure the wavefront effects of each poke. This combination of poke pattern data leads to a sensitivity matrix which is later used by APRC to convert the measured MGS wavefront error into DM voltages.

The MGS influence procedure can be affected by high dynamic range phase wrapping at the location of the DM pokes where low order aberrations in the wavefront are already unusually high. To reduce this effect, several influence measurement trials at various DM poke heights $(300,275,250,225,200 \mathrm{~nm})$ were completed. With the DM pokes set to $250 \mathrm{~nm}$, no phase wrapping occurred during the measurements. This $250 \mathrm{~nm}$ data set also produced the best results during the week that lab tests were done, and so this sensitivity matrix is the standard for APRC going forward.

During influence measurement testing it became clear that the APRC/MGS results are strongly coupled to air turbulence in the optical path. Some of this turbulence was isolated to air currents in the lab, and so then reduced by covering the top of the exposed stimulus side of the PALAO bench. But some of the turbulence is still present in the optical path, and is not controllable. As a result, longer PHARO exposure times were tried, both for the standard APRC science exposures and for the exposures taken for these influence measurements. In the lab (typically $70^{\circ} \mathrm{F}$ ) the best results were obtained using exposure times of 30 seconds.

When an APRC iteration is completed, the result is displayed on the users screen which shows both the total measured NCP wavefront error and the "controllable" wavefront error. The controllable wavefront error is simply the total error passed through a low-pass filter at 8 cycles per pupil, and is meant to convey the residual error that the DM should be able to correct (residual errors at frequencies higher than 8 cycles per pupil are not controllable by the DM). At first glance, a typical "best-case" APRC controllable error appears to show small bumps and valleys that look similar in size to a DM actuator, suggesting that actuators are not being controlled as well as desired. But when a map of the actuator positions that APRC determined during the influence measurements is overlaid, it's clear that most of the controllable residual error in the inner $80 \%$ of the pupil exists between actuators. It's also clear that the centroiding algorithm that APRC uses to locate actuator pokes has trouble with poorly illuminated actuators at the outer pupil boundary, which may explain why the controllable error is higher in this region. This issue has not yet been addressed. 


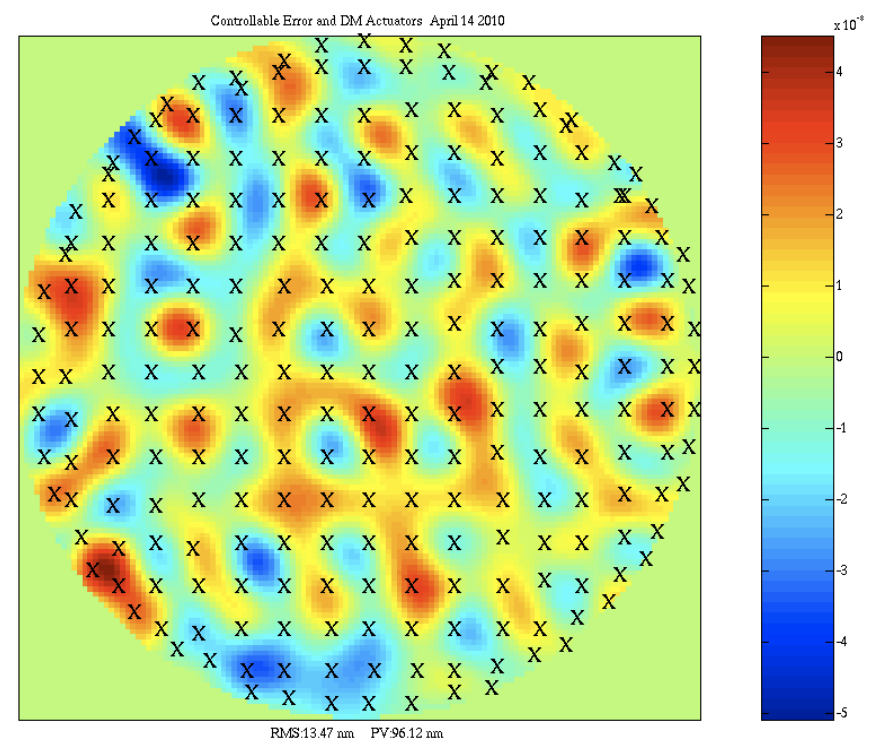

Figure 4. APRC Controllable Error: A best case APRC result from April 2010. The total APRC NCP wavefront error is passed through a low-pass filter at 8 cycles per pupil, showing what the PALAO DM should be able to correct. Overlaid on this residual error are the actuator positions that APRC determined during influence measurements. Note that most of the residual error in the central $80 \%$ of the pupil exists between DM actuators.

\subsection{CLOSING THE LOOP WITH APRC}

PALAO uses a Shack Hartmann sensor to measure the centroids of the stellar target spots in each of the sensor subapertures whose centroid locations are determined by the incoming wavefront. The global grid of centroids is then used to reconstruct the measured wavefront. Centroid offsets are numbers subtracted from the measured centroids before the wavefront is reconstructed. The centroid offsets are in fact the values used to correct for NCP wavefront errors, and had been up to this point generated entirely by a manual hand tuning method, which for best case resulted in $110 \mathrm{~nm}$ residual wavefront error. To extend the APRC results from DM actuator voltages to something that can be used on sky, the APRC solution is applied to the DM. Centroid offsets are then all set to zero, and the high order loop is locked for 30 seconds. During this time the PALAO wavefront sensor camera measures the centroids generated by the APRC solution. These centroids are then extracted, averaged, and copied into a new centroid offset file. With this new centroid offset file loaded and the high order loop closed, the DM is now being commanded to the APRC solution, and APRC is now ready to go to the sky.

As local seeing conditions at Palomar change, the spot size of the target star in the wavefront sensor also changes. As the spot size increases as seeing gets worse, PALAO performance may degrade when centroids are driven far from zero. High order performance can be recovered in most cases by using centroid offsets that have been divided by 2 , and in some cases 4 or 6 , such that the centroid slopes are retained but the amplitude of the deviations are reduced. APRC now generates these additional centroid offset files, which can be quickly loaded by the PALAO operator, and then verified by the observer before target observations begin.

\subsection{TURBULENCE IN PALAO}

The APRC/MGS solutions depend strongly on air turbulence and perhaps air temperatures in and around the PALAO bench when the APRC measurements are taken. The effect of this turbulence can be minimized in the lab by tightly covering the top of the optical bench and turning the lab air conditioning blowers off. But the turbulence is not removed 
completely, suggesting that some of the air currents in the PALAO bench cannot be controlled. Tests that tried to isolate hardware components to determine air current sources in the optical bench were inconclusive.

The APRC solution is a measurement of the difference of two defocused images. By default APRC commands the PHARO science camera to take as fast an exposure as possible (1.4 seconds) in order to reduce the total time it takes to finish an iteration. To better determine the proper exposure time necessary to reduce the air turbulence effects, long sets of open loop data were taken with the PALAO wavefront sensor looking at the white light source. DM residual error wavefronts were then extracted from this long data set. Using an approach very similar to an Allan variance, averages of identical exposure times were subtracted over the entire DM residual error data set (starting with one second). Plots were then constructed of the residual wavefront errors (rms) from these differences against the exposure time.

At the time of these tests the best controllable error from the APRC solutions was $15 \mathrm{~nm} \pm 5 \mathrm{~nm}$ (rms). In the typical lab environment with tight covers on the optical bench, the $15 \mathrm{~nm}$ residual error level was reached in 30 seconds. APRC was then adjusted to command PHARO to take 30 second exposures at that time, and this has been the standard exposure time used for APRC. Similar data sets were collected when on the telescope in ambient temperatures of $19^{\circ} \mathrm{C}$ (similar to the lab, but without tight covers on the PALAO stimulus) and $2^{\circ} \mathrm{C}$. The plots indicate that exposure times closer to 60 seconds are required to reach the $15 \mathrm{~nm}$ residual error level when PALAO is on the telescope, likely because the tight cover over the stimulus must be removed when PALAO is installed. Further, when PALAO is exposed to very cold air as is typically the case in the winter months, these plots suggest that air turbulence is so strong that exposure times approaching 200 seconds are required to reduce the residual errors to the $15 \mathrm{~nm}$ level.

The entire APRC procedure now takes 5 minutes per iteration; 2.5 minutes to automatically command PALAO and PHARO to obtain the two defocused images, and 2.5 minutes to automatically send the data to JPL, commence the MGS processing, and return the results to the PALAO control computer at Palomar. The entire procedure is now done via one command issued from a MATLAB session on the PALAO control computer. From day to day, three iterations are typically required to get to the noise floor when APRC is run in the lab or on the telescope if the dome air temperatures are above $10^{\circ} \mathrm{C}$. The APRC/MGS noise floor in these cases is $13 \mathrm{~nm} \pm 3 \mathrm{~nm}(\mathrm{rms})$. Once the APRC floor is reached, one iteration is usually required to recover $\mathrm{NCP}$ wavefront error when the telescope position is changed.
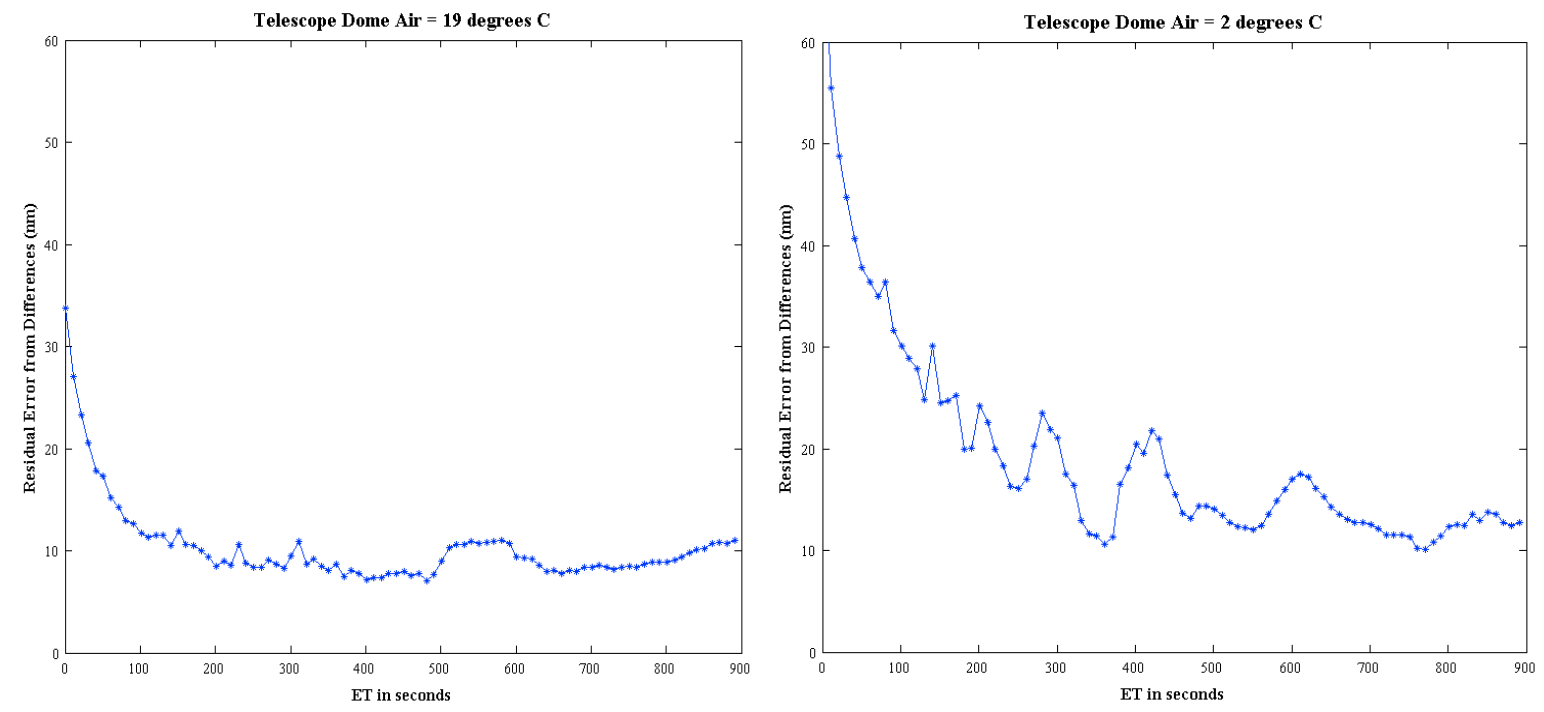

Figure 5. Air turbulence in the PALAO bench: left. Plot of the mean residual errors of differenced data sets against the exposure time used for that data set in the PALAO bench when installed on the telescope. The dome air temperature for this plot was $66^{\circ} \mathrm{F}$. This plot suggests that 60 seconds is the ideal exposure time to reach the $15 \mathrm{~nm}$ residual error level. right. The same plot but with the dome air temperature at $35^{\circ} \mathrm{F}$. This plot suggests that at least 250 seconds is the ideal exposure time to reach the $15 \mathrm{~nm}$ residual error level. Strong turbulence from warm surfaces in the optical bench and DM actuator performance in the cold temperatures are possible contributors to the poorer performance. 


\subsection{APRC FOCUS CONTROL}

When APRC was first implemented in 2007, focus was allowed to pass into the APRC solution and so was propagated to the DM. In addition the measured focus in the solution was converted to linear stage units and printed to the screen so a user could move the white light internal stage to optimize the white light focus position before the next iteration. This was considered adequate as long as care was taken to make sure PHARO was focused manually (using a different procedure) before running APRC. To improve on this, the APRC focus solution is now used to automatically move both the white light stage and the PALAO wavefront sensor camera stage in equal but opposite amounts to effectively remove focus from the APRC solution, and keep focus off of the DM. These new motor stage positions are saved to the PALAO system settings so that they are always used if the system is stopped.

It is important to point out that MGS is an image tuning algorithm, which means these methods are used to fine tune wavefront errors measured at the image plane of the detector. MGS does not measure wavefront errors at the PHARO focal plane where the coronagraph is located. High contrast performance requires superb wavefronts at the coronagraph, and so to benefit from MGS, the PHARO optical quality between the entrance focal plane and the final image plane must be as good as the PHARO design specifications say it is. To test this, the internal white light source is centered behind the VVC mask and small amounts of low order Zernike's are applied to the DM. The images are then measured for any changes to contrast. No measureable change to the contrast level was seen, verifying the excellent quality in the optical path between the PHARO focal and image planes.

However, a focus offset does exist between the true PHARO focal plane and the position of the VVC phase mask, which was inserted into a clear substrate and mounted into the PHARO focal plane selector mechanism. It was suspected that the mask is not located at the true focal plane, and so to test this, the PALAO internal white light source was positioned behind the VVC mask, and images were taken in PHARO's pupil imaging mode. A ring of scattered light illuminating the outer edge of the pupil images was immediately seen, which is expected if the mask is not perfectly located in the PHARO focal plane. The PALAO wavefront sensor stage is translated until the pupil image is nicely dark. The offset between the VVC and the PHARO focal plane is corrected by a $700 \mu \mathrm{m}$ translation of the PALAO wavefront sensor stage, and so this focus value is added to the stage after the APRC floor is reached.

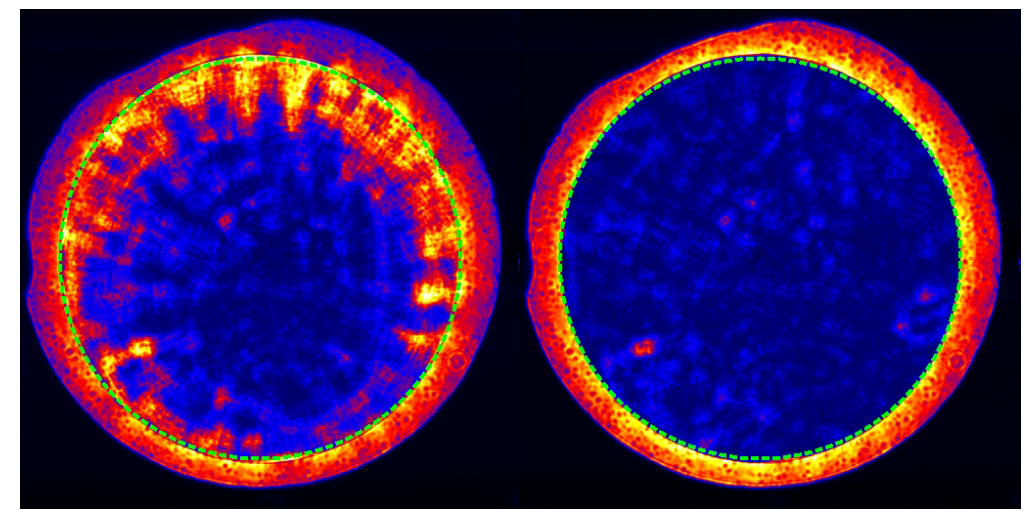

Figure 6. Before and after correcting the Vector Vortex focus offset: left. PHARO pupil image with the internal PALAO white light source centered behind the VVC mask and the APRC solution loaded onto the DM. The wavefront entering PHARO is not correctly focused on the VVC mask, so some of the light is not blocked and is seen at the outer boundary of the pupil image. The green dashed line is the outer edge of the PALAO pupil. right. PHARO pupil image after translating the PALAO wavefront sensor stage 700 microns. The light from the source is now perfectly focused on the VVC mask. 


\subsection{APRC ON THE TELESCOPE}

In February 2010 an APRC iteration was completed at a non-zenith telescope position which measured a $40 \mathrm{~nm} \mathrm{rms}$ increase in the wavefront error, suggesting that the total wavefront error changed simply by moving the telescope. At that time it was not known how much of the $40 \mathrm{~nm}$ increase was in the common path to both PALAO and PHARO, which would then be corrected by PALAO, or how much of this error was only in the NCP, which would not be corrected by PALAO.

In April 2010 a test was conducted to measure wavefront errors induced by non-zenith telescope pointings in such a way as to separate the common and non-common components. This was started by running APRC on the white light at zenith to establish the reference wavefront error, measured at $22 \mathrm{~nm}$ rms. The APRC solution was copied into the reference PALAO flatmap (a map of actuator commands that leave the DM in the best possible open loop shape to create the best science image). Centroid offsets were also generated on this map.

The telescope was slewed to various telescope positions, starting in the east and slewing west, for each chosen declination. At each telescope pointing one iteration of APRC was run on the white light, which measures the wavefront error through PALAO up to the science dichroic AND everything after the dichroic, including PHARO. This measurement is the "total" error.

The high order loop is then closed on the white light (using the reference centroid offsets) and PALAO then records a new flatmap. The difference of this flatmap to the reference flatmap taken at zenith measures the wavefront error through PALAO (as above) and everything reflected off of the science dichroic (the PALAO wavefront sensor arm). Assuming that the wavefront error through the wavefront sensor arm is small, this measurement is the "common path" error.

The difference of the total error and the common path error is the non-common path error that is present at that telescope position. For most telescope positions within $30^{\circ}$ of zenith, the controllable residual NCP wavefront error is greater than $20 \mathrm{~nm} \mathrm{rms}$. And for telescope pointings beyond $30^{\circ}$ of zenith, the residual NCP wavefront error is typically greater than $50 \mathrm{~nm} \mathrm{rms}$. This is due to gravity loads effecting optical mounts in the science arm. So to achieve the highest contrast possible at any non-zenith telescope pointing, APRC needs to be run at least once with the telescope pointed at the target location before observing. Note that APRC is always run on the internal white light source, it is not run on stars where the changing atmosphere adds phase errors to the raw data that skew the MGS result.
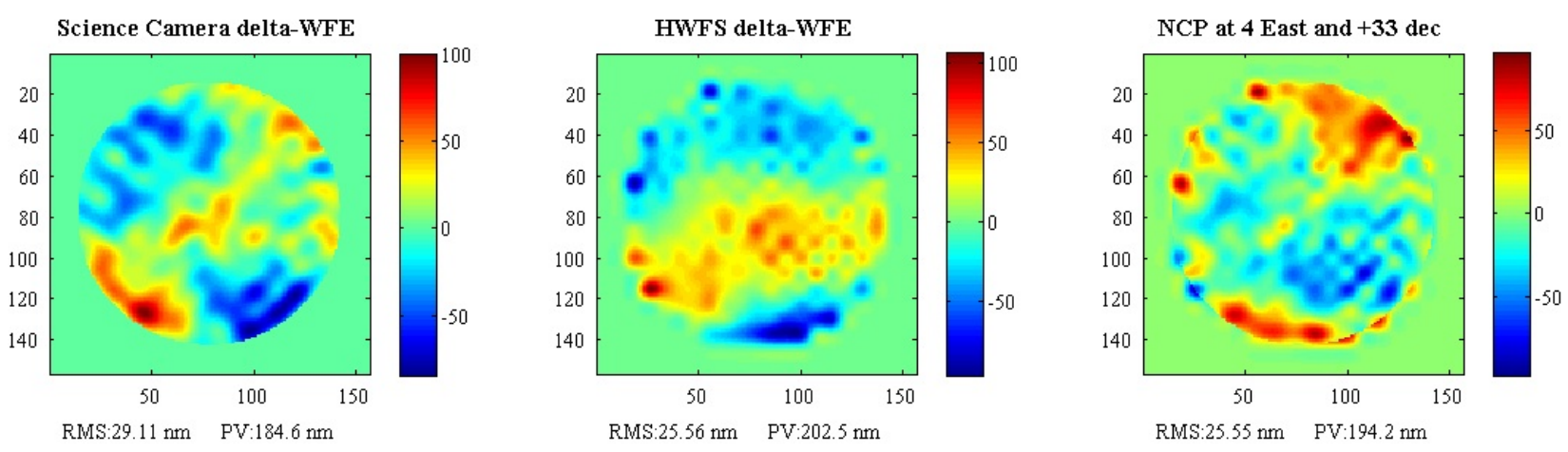

Figure 7. Wavefront errors with the telescope pointed to an hour angle of 4 hours East and a declination of $+33^{\circ}$ : left. The APRC solution measures a controllable difference of $29 \mathrm{~nm}$ rms between zenith and this telescope position. This is the total error in the system. middle. The PALAO wavefront sensor solution measures a common path difference of $25 \mathrm{~nm}$ rms. right. The difference of these two measurements is the non common path error and is also $25 \mathrm{~nm}$ rms in this case. These images show that the errors induced by changing telescope positions are low order in nature, and so correctable by the DM. This strongly implies that APRC should be run at the beginning of each telescope position if high contrast performance is required. 


\subsection{APRC TODAY}

APRC is now available to the entire PALAO observing community. The entire procedure is run by a single command executed on the PALAO control computer by the Palomar staff. It is a robust program that gives all PHARO users an on-sky NCP wavefront quality improvement from $110 \mathrm{~nm}$, which is the best that can be generated by the hand tuning method, to less than $40 \mathrm{~nm}$ rms. APRC is also efficiently quick to provide wavefront improvements at various telescope positions for observers who require the absolute best corrections of the non-common path errors. The typical residual wavefront errors left over when APRC is done are remarkably low: $35 \pm 4 \mathrm{~nm}$ rms total wavefront error, where only $13 \pm$ $3 \mathrm{~nm}$ rms remains in the controllable pass band of the deformable mirror.
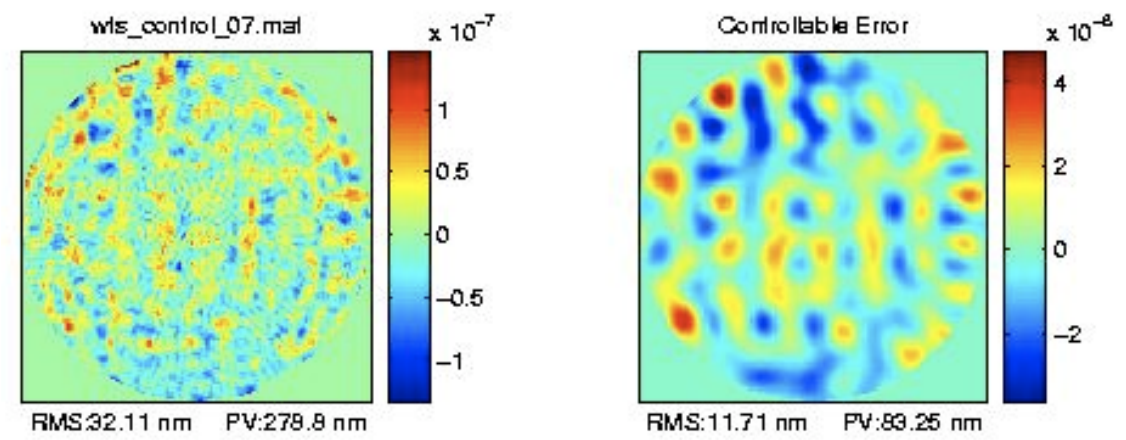

Figure 8. Typical APRC best case result: left. Usually after only 3 iterations, APRC reaches a total error of $35 \pm 4 \mathrm{~nm}$ rms right. The best case controllable error is $13 \pm 3 \mathrm{~nm}$ rms.

\section{ON SKY RESULTS}

In the spring and summer of 2009 WCS, PALAO, PHARO and APRC were joined together with the improved performance of the PALAO pointing mounts and taken to the sky for the first time. In July 2009, HR8799 was observed. For these observations APRC was run during the evening just before opening the dome, but it was not run at each target. We immediately noticed improved contrast in the raw VVC images, even though the seeing was a modest 1.6 arcseconds.

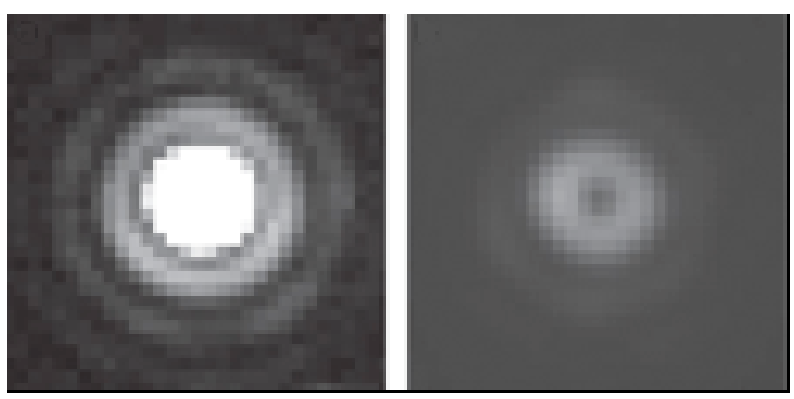

Figure 9. Pre and post-vortex HR8799 point spread function: left. The Ks-band raw stellar point spread function taken on July 11, 2009 with the WCS in 1.6" seeing. Strehl ratio is $0.91 \pm 0.008 \mathrm{rms}$. right. The post vector vortex coronagraph point spread function on the same intensity scale. The peak to peak stellar rejection ratio is approximately 50, consistent with the observed Strehl ratio. Both images were taken with the same APRC solution applied to the high order correction. This APRC solution was acquired with the telescope pointing at zenith earlier in the evening. 
Calibrator stars of similar magnitude and color to the target are also observed, to be used as a reference to subtract the speckle noise from the target images. To do this effectively, the speckles need to remain constant between the target and the calibrator. Calibrators that are relatively close to the target star are chosen so that flexure does not introduce unwanted speckle noise in the images. As the telescope tracks across the sky, contrast is ultimately limited to the time it takes for the speckles to change significantly enough such that calibrator subtraction is no longer effective. Data taken on HR8799 and other high contrast sources independently verifies that a full magnitude of contrast is lost in about 8 minutes, strictly due to speckle decorrelation. For this reason efficient telescope slew techniques are now used to rapidly switch between targets and calibrators.

\subsection{POST PROCESSED RESULTS}

Due to the excellent stability and performance provided by the WCS, the new image pointing mounts, and the APRC solution, the quality of the raw data was so good that nearly a full order of magnitude improvement in contrast was achieved using the "locally optimized combination of images" (LOCI) algorithm" to reference subtract calibrator stars from the target. In the case of HR8799, where both the target and two calibrator stars were observed for 800 seconds each, the fully reduced image shows all 3 known exoplanets clearly, including the innermost "d" planet whose separation from the host star is only $2 \lambda / \mathrm{D}$ (about $550 \mathrm{mas}$ in this case). The APRC enhancements alone led to an order of magnitude improvement in the residual speckle noise when combined with the VVC. From the HR8799 data set, the post-VVC semi-static speckle noise at the $4 \sigma$ level shows to a contrast of $1 \times 10^{-4}$ at $2.5 \lambda / \mathrm{D}$ separations. After running the LOCI algorithm on the data sets, contrast ratios of nearly $2 \times 10^{-5}$ at the $4 \sigma$ detection limit are achieved all the way in to $\lambda / \mathrm{D}$ separations, which is within a factor of two of the photon noise expected in the coronagraphic images.

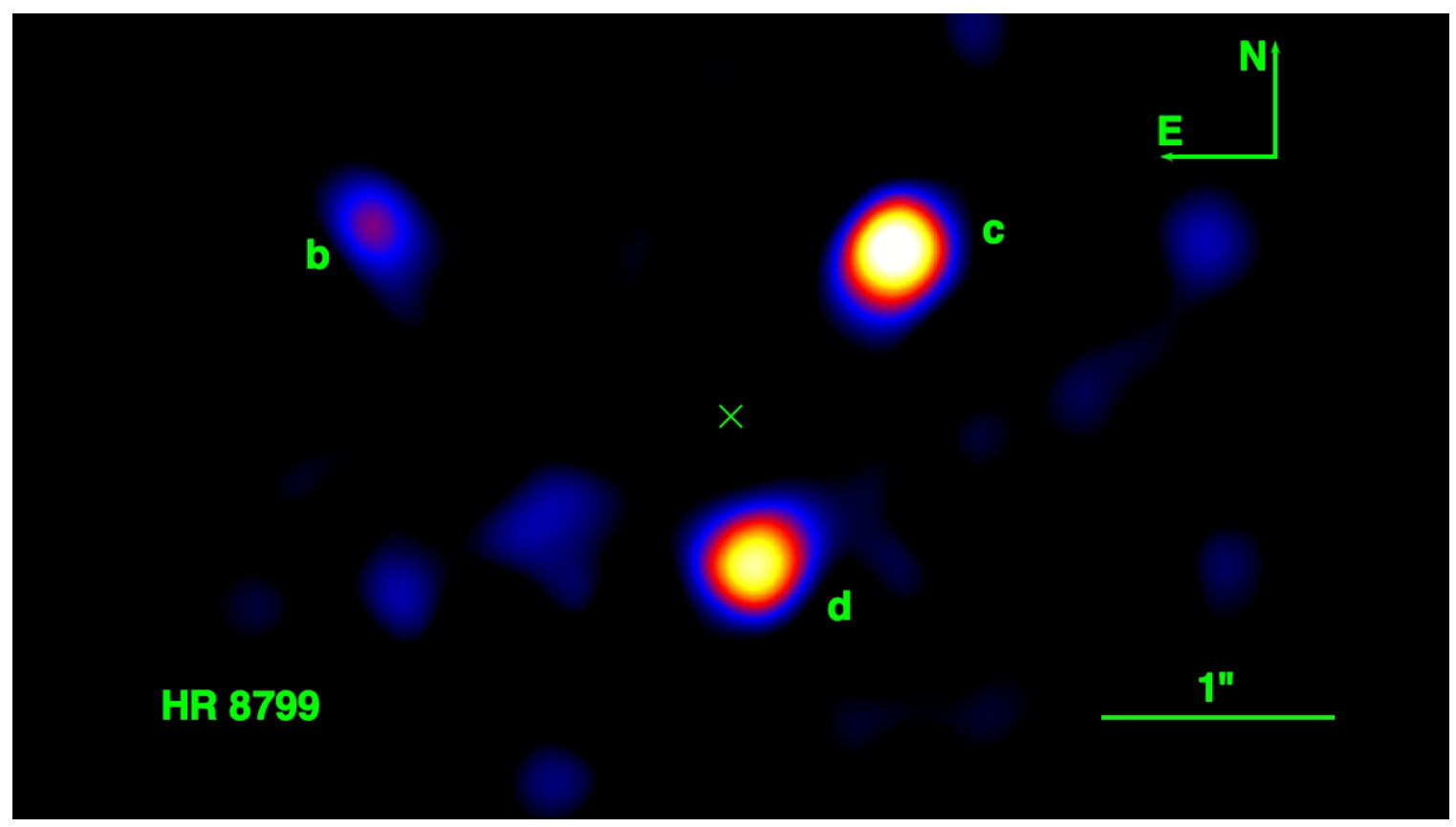

Figure 10. Final reduced image of HR8799: The fully reduced HR8799 exoplanet system after reference star subtraction using the LOCI algorithm. The innermost "d" exoplanet is located $2 \lambda / \mathrm{D}$ away from the center of the star. The central $1 \lambda / \mathrm{D}$ region is blanked out. 
Our 2009 data sets are limited by the speckle decorrelation time (the time it takes for speckles to change significantly at and between targets) and NCP residual errors induced by changing telescope positions. To minimize these problems, efficient observing techniques were incorporated in 2010 to rapidly slew between target and calibrator stars. In addition, APRC is now run at each target position before observing, ensuring that the residual NCP errors in the controllable band are below $20 \mathrm{~nm}$ rms for the observations. The star Epsilon Eridani was observed in 2010 using these new techniques, and the contrast achieved in these new images is remarkable. The post-VVC semi-static speckle noise at the $1 \sigma$ level, which shows what the APRC solution coupled to the WCS and VVC can achieve, is measured at $5 \times 10^{-5}$ at less than 1" separations before post-processing techniques. After LOCI processing, contrasts of $2 \times 10^{-6}$ are achieved at the $1 \sigma$ level at less than 1" separations.

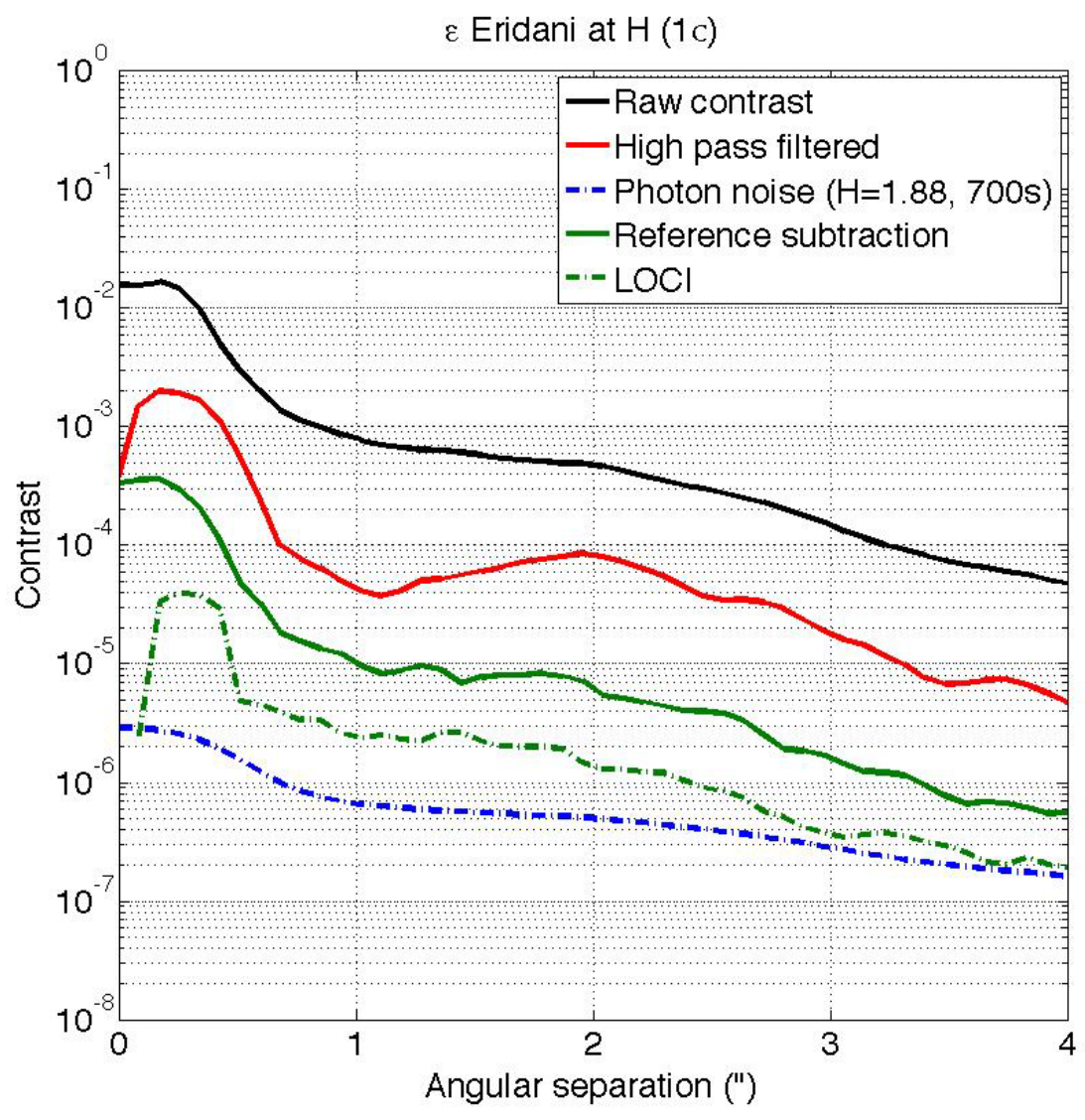

Figure 11. Epsilon Eridani point spread function profiles and limiting contrast curves: The top solid curve is the azimuthally averaged coronagraphic point spread function profile. The next solid curve (red) shows the radial dependence of the post-VVC semi-static speckle $1 \sigma$ noise level after using the APRC solution. This shows that WCS, VVC, and APRC alone reach the $5 \times 10^{-5}$ contrast level at less than 1 " separations. The next solid curve (green) shows the azimuthally averaged $1 \sigma$ detection limit after a standard reference start subtraction. The next dashed curve (green) shows the azimuthally averaged $1 \sigma$ detection limit after a reference start subtraction using LOCI. While the LOCI solution is obviously better, both methods, given the excellent pre-reduction performance, have reached the $10^{-5}$ and $10^{-6}$ contrast levels. 


\section{FUTURE WORK}

The VVC inner working angle is currently limited by residual high frequency tip tilt errors, which produces a halo around the center of the VVC mask. Attempts to reduce these errors by manipulating the PALAO tip tilt servo gains will be done during the next observing run. An automated VVC centering algorithm will be implemented to monitor the raw coronagraphic PHARO images and adjust the PALAO image pointing to keep targets centered on the masks.

In the fall of 2010 the PALAO bench will be temporarily decommissioned for 6 months to upgrade to a 3000 actuator deformable mirror $(\mathrm{P} 3 \mathrm{~K})^{10}$. APRC will be adapted to work with P3K, and MGS will be run on a spare P3K graphics card locally at Palomar. Testing MGS on this card shows that the MGS algorithm can be run in 6 seconds, rather than the 2 minutes that it takes now.

\section{ACKNOWLEDGEMENTS}

This work was carried out at the Jet Propulsion Laboratory, California Institute of Technology, under contract with the National Aeronautics and Space Administration, and is based on observations obtained at the Hale Telescope, Palomar Observatory, as part of a continuing collaboration between the California Institute of Technology, NASA/JPL, and Cornell University. We thank the staff of the Palomar Observatory and the Palomar Adaptive Optics team for their continued support.

Reference herein to any specific commercial product, process, or service by trade name, trademark, manufacturer, or otherwise, does not constitute or imply its endorsement by the United States Government or the Jet Propulsion Laboratory, California Institute of Technology.

\section{REFERENCES}

[1] S. Bikkannavar, C. Ohara, M. Troy, "Autonomous phase retrieval control for calibration of the Palomar adaptive optics system", Proc SPIE, Vol. 7015, 70155K, (2008)

[2] J. Green, D. Redding, S. Shaklan, S. Basinger, "Extreme Wavefront Sensing Accuracy for the Eclipse Coronagraphic Space Telescope", Proc. SPIE, Vol. 4860, 266-276, (2003)

[3] C. Marois, et. al., "Direct imaging of multiple planets orbiting the star HR8799", Science 322, 1348-1352, (2008).

[4] E. Serabyn, et. al., "Extreme adaptive optics using an off-axis subaperture on a ground-based telescope", Proc SPIE, Vol. 6272, 62722W, (2006).

[5] E. Serabyn, et. al., "An off-axis four-quadrant phase-mask coronagraph: concept and first results", Direct Imaging of Exoplanets: Science \& Techniques. Proceedings of the IAU Colloquium \#200, 477-480, (2006)

[6] D. Mawet, et. al., "The Vector Vortex coronagraph: laboratory results and first light at Palomar Observatory", Astrophysical Journal 709, 53-57, (2010).

[7] E. Serabyn, D. Mawet, R. Burruss, "An image of an exoplanet separated by two diffraction beamwidths from a star", Nature, 464, 1018-1020, (2010)

[8] Aerotech, Inc., Pittsburgh, PA, United States, Phone: 1-412-963-7470, www.aerotech.com

[9] D. Lafreniere, et. al., "A new algorithm for point-spread function subtraction in high-contrast imaging: a demonstration with angular differential imaging", Astrophysical Journal 660, 770-780, (2007).

[10] A.H. Bouchez, et. al., "The PALM-3000 high-order adaptive optics system for the 5.1m Hale Telescope", Proc. SPIE, Vol. 7015, 70150Z, (2008). 